\title{
P-glycoprotein associated with diabetes mellitus and survival of patients with pancreatic cancer: 8-year follow-up
}

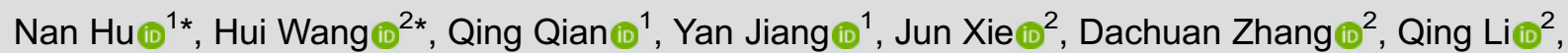 \\ Sulan Zou (10 ${ }^{1}$, and Rong Chen ${ }^{1}{ }^{1}$ \\ ${ }^{1}$ Department of Pharmacy, The Third Affiliated Hospital of Soochow University, Changzhou, Jiangsu, China \\ ${ }^{2}$ Department of Pathology, The Third Affiliated Hospital of Soochow University, Changzhou, Jiangsu, China
}

\begin{abstract}
Diabetes mellitus (DM) has a high prevalence in patients with pancreatic cancer $(\mathrm{PaC})$, but the prognostic value of $\mathrm{DM}$ in $\mathrm{PaC}$ remains controversial. Alterations of P-glycoprotein (P-gp) and cytochrome P450 3A4 (CYP3A4) contribute to multidrug resistance and intestinal metabolism in a variety of cancer types, which may be implicated in DM development. This study aimed to explore the potential prognostic value of P-gp and CYP3A4 in PaC patients in the context of DM through long-term follow-up. We retrospectively reviewed the medical records of patients with $\mathrm{PaC}$ admitted at The First People's Hospital of Changzhou, Jiangsu, China, from January 2011 to November 2019 and identified two cohorts of adult patients with PaC, including 24 with DM and 24 without DM (non-DM). The baseline clinical characteristics and outcomes were compared. Immunohistochemistry showed that protein expression of P-gp, but not CYP3A, in duodenum tissues was significantly upregulated in $\mathrm{PaC}$ patients with DM compared with those without DM. Kaplan-Meier analysis and log-rank test showed that the survival of patients with $\mathrm{PaC}$ and $\mathrm{DM} /$ high expression of P-gp was not significantly reduced compared with that of patients without DM/low expression of P-gp. These findings suggested that P-gp expression levels were different in the DM and non-DM groups of patients with $\mathrm{PaC}$, but $\mathrm{DM}$ and duodenal $\mathrm{P}$-gp levels were not associated with the long-term survival of patients with $\mathrm{PaC}$. It appears that the presence of DM or P-gp expression levels may not serve as effective prognostic markers for $\mathrm{PaC}$.
\end{abstract}

Key words: Cohort study; Cytochrome P450 3A4; P-glycoprotein; Pancreatic cancer with diabetes mellitus

\section{Introduction}

According to GLOBOCAN 2018, pancreatic cancer $(\mathrm{PaC})$ is the 11th most common cancer, estimated to have caused 432,242 deaths in 2018 (1). Currently, available treatment options for $\mathrm{PaC}$ are surgical resection, chemotherapy, and radiotherapy (2), among which surgical resection is considered the mainstay of curative treatment and is associated with better long-term survival (3). Nevertheless, $80-90 \%$ of patients with $\mathrm{PaC}$ are diagnosed at an advanced stage and with unresectable tumors, and the 5 -year survival rate of $\mathrm{PaC}$ is as low as $5 \%$ (4). Identification of poor prognostic factors in $\mathrm{PaC}$ may provide valuable prognostic information for selecting appropriate treatment protocols. Although factors like tumor stage, grading, lymph node ratio, surgical margin, and tumor size have been identified as prognostic factors in $\mathrm{PaC}(5,6)$, it is of great importance to identify new biological or pathological indicators related to the survival of patients with $\mathrm{PaC}$.

Diabetes mellitus (DM) is a group of metabolic disorders characterized by hyperglycemia resulting from insufficient insulin secretion, resistance to insulin action, or both (7), posing an increasingly serious threat to human health worldwide with an estimated global prevalence of 592 million by 2035 (8). DM is known as a possible risk factor and/or an early manifestation of $\mathrm{PaC}$, being concurrently present in $50-80 \%$ of patients with $\mathrm{PaC}(9,10)$. Accumulating evidence suggests that concurrent diagnosis of DM is associated with increased risk of cancer recurrence and mortality in patients with colorectal, breast, liver, endometrial, and gastric cancers as well as leukemia (11). Nevertheless, how DM affects the clinical outcome and survival of patients with $\mathrm{PaC}$ remains controversial. Some studies

Correspondence: Rong Chen: <pharmacy_czyy@163.com>

${ }^{*}$ These authors contributed equally to this work.

Received April 12, 2020 | Accepted July 17, 2020 
reported that the presence of DM does not have a statistically significant effect on the overall survival (OS) or the mortality of $\mathrm{PaC}(11,12)$, whereas others found a significant association between DM and reduced survival in $\mathrm{PaC}$ (13). Therefore, the characterization of a reliable indicator related to survival and clinical outcome is required for predicting prognosis and selecting appropriate therapeutic strategies for patients with $\mathrm{PaC}$ and $\mathrm{DM}$.

Systemic chemotherapy is used for advanced $\mathrm{PaC}$ after surgery or unresectable metastatic $\mathrm{PaC}$, which significantly extends OS and improves patient outcome (14). Intrinsic or acquired resistance to chemotherapy remains the major cause of treatment failure (15). The drug efflux protein P-glycoprotein (P-gp) and drug-metabolizing enzyme cytochrome P450 3A4 (CYP3A4) are both expressed in the intestinal mucosa and serve as barriers to oral drug delivery by regulating pharmacokinetic and pharmacodynamic interactions during the process of drug absorption and metabolism $(16,17)$. P-gp and CYP3A4 are overexpressed in a number of different cancers such as ovarian, breast, and colon cancers, and some studies showed that they are associated with resistance to therapy and/or poor prognosis $(18,19)$. In patients with coexisting $\mathrm{PaC}$ and $\mathrm{DM}$, alterations of P-gp and CYP3A4 may become more complicated due to the hyperglycemic environment and diabetes medications (20). A recent study showed that the expression of intestinal P-gp was significantly increased with the progression of diabetes in rats (induced by low dose streptozotocin and high-fat diet), resulting in a significant decrease in the intestinal uptake and peroral bioavailability of the P-gp/CYP substrates verapamil and atorvastatin (21). Peroral bioavailability of drugs is a primary concern for patients with coexisting $\mathrm{PaC}$ and $\mathrm{DM}$ who are usually prescribed with multiple drugs for effective management of blood glucose levels and cancer progression. Little is known with respect to the expression patterns of intestinal P-gp and CYP3A4 in PaC patients in the context of $\mathrm{DM}$, as well as their correlation with patient prognosis.

In the present study, we retrospectively reviewed the records of patients with $\mathrm{PaC}$ admitted to The First People's Hospital of Changzhou, Jiangsu, China from January 2011 to November 2019, and identified 24 patients with $\mathrm{PaC}$ and $\mathrm{DM}$ and 24 with $\mathrm{PaC}$ but without $\mathrm{DM}$. The protein expression of P-gp and CYP3A4 in the duodenum tissue of each patient was determined. The association between $\mathrm{P}$-gp/CYP3A expression and the presence of DM as well as between survival time and DM presence/P-gp expression were assessed. Our findings may provide a new understanding of the value of intestinal P-gp expression in evaluating the prognosis in $\mathrm{PaC}$ patients with $\mathrm{DM}$.

\section{Material and Methods}

\section{Patients}

From the database of patients at The First People's Hospital of Changzhou, Jiangsu, China, from January
2011 to November 2019, we identified 24 consecutive adult patients with pathologically confirmed $\mathrm{PaC}$ and $\mathrm{DM}$. Another cohort of unmatched 24 patients with $\mathrm{PaC}$ but without DM (non-DM) was randomly selected from the database. The patients with other cancers, undergoing radiotherapy/ chemotherapy, with incomplete data, or lost to follow-up were excluded. The patient characteristics are described in Table 1. This study was approved by the Ethics Committee of The First People's Hospital of Changzhou. Informed consent was obtained from each patient.

\section{Immunohistochemistry (IHC)}

IHC was performed using the EliVision ${ }^{\mathrm{TM}}$ method (Maixin-Bio, Fuzhou, China). Duodenum tissue samples were obtained during surgery and fixed in $4 \%$ formalin. Paraffin-embedded tissue sections (3-4- $\mu \mathrm{m}$ thick) were prepared. The sections were dewaxed in xylene and dehydrated in ethanol, followed by incubation with $3 \%$ $\mathrm{H}_{2} \mathrm{O}_{2}$ for 15 min. After additional incubation with $10 \%$ normal bovine serum for $10 \mathrm{~min}$, each slide was incubated with primary antibodies against human P-gp (MAB-0237, MXB Biotechnologies, China) or CYP3A4 (ab3572, Abcam, UK) at $4^{\circ} \mathrm{C}$ overnight. The protein expression was visualized using an ultraView universal DAB detection kit (Ventana, Roche, USA). The positive and negative controls were provided by the manufacturer. The results were blindly scored (0-100\%) by two independent pathologists using an Olympus IX73 microscope (Olympus Corp., Japan) and the following algorithm: [ $(3 \times$ intensity of specific immunodetection) $+(2 \times$ amount of immunodetected structures $)+(2 \times$ intensity of non-specific immunodetection $)+(1 \times$ intensity of contrast $)+(1 \times$ preservation of morphology $)] \times$ 3703 (constant that allows conversion to the 0-100 score), as previously described (22). Five randomly selected fields were scored in each slide. The final score is reported as the average of the scores of two pathologists. A reassessment was performed when the deviation was $\geqslant 20 \%$.

\section{Statistical analysis}

Statistical analyses were carried out using the SPSS software (version 19.0; IBM, USA). Data are reported as median (range). Comparison between two groups was conducted using the chi-squared test or unpaired Student's $t$-test. Differences between categorical variables were compared using the chi-squared test. The survival of patients with $\mathrm{PaC}$ was assessed using Kaplan-Meier analysis and the log-rank test. $A$ value of $P<0.05$ was considered statistically significant.

\section{Results}

\section{Comparison of clinicopathological characteristics between patients with $\mathrm{PaC}$ with/without DM}

To identify the possible features of DM in patients with $\mathrm{PaC}$, we compared the clinicopathologic characteristics of patients with $\mathrm{PaC}$ with/without DM. In the DM group, the 
Table 1. Clinicopathological characteristics of patients with pancreatic cancer with or without diabetes mellitus (DM).

\begin{tabular}{|c|c|c|c|}
\hline Clinicopathological variables & $\mathrm{DM}(\mathrm{n}=24)$ & Non-DM $(n=24)$ & $P$ \\
\hline Sex & & & 0.019 \\
\hline Male & $10(41.7 \%)$ & $18(75.0 \%)$ & \\
\hline Female & $14(58.3 \%)$ & $6(25.0 \%)$ & \\
\hline Age & $67.50(43.00,83.00)$ & $65.00(48.00,82.00)$ & 0.297 \\
\hline Weight (kg) & $59.00(45.00,78.00)$ & $62.00(46.00,89.00)$ & 0.260 \\
\hline Height $(\mathrm{cm})$ & $161.50(152.00,175.00)$ & $166.50(156.00,177.00)$ & 0.032 \\
\hline Smoking/alcohol & $1(4.3 \%)$ & $3(12.5 \%)$ & 0.317 \\
\hline Hypertension* & $9(37.5 \%)$ & $9(37.5 \%)$ & 1.000 \\
\hline Duration of DM (years) & $3.00(0.10,30.00)$ & - & $\mathrm{N} / \mathrm{A}$ \\
\hline Medications & & & $\mathrm{N} / \mathrm{A}$ \\
\hline N/A & $2(11.8 \%)$ & - & \\
\hline Gliclazide & $1(5.9 \%)$ & - & \\
\hline Metformin & $1(5.9 \%)$ & - & \\
\hline Metformin + repaglinide & $2(11.8 \%)$ & - & \\
\hline Metformin + insulin & $1(5.9 \%)$ & - & \\
\hline Repaglinide & $2(11.8 \%)$ & - & \\
\hline Insulin & $8(47.1 \%)$ & - & \\
\hline Fasting blood glucose (mM) & $7.95(3.80,12.50)$ & $5.70(3.90,12.30)$ & 0.001 \\
\hline Postprandial blood glucose (2 h, mM) & $14.90(6.90,28.50)$ & - & $\mathrm{N} / \mathrm{A}$ \\
\hline WBC count $\left(\times 10^{9}\right)$ & $5.86(3.99,11.30)$ & $5.27(3.07,10.75)$ & 0.068 \\
\hline RBC count $\left(\times 10^{12}\right)$ & $3.94(2.70,5.18)$ & $4.36(2.41,5.41)$ & 0.155 \\
\hline Hemoglobin $(g / L)$ & $124.50(86.00,160.00)$ & $132.50(65.00,149.00)$ & 0.458 \\
\hline ALT (U/L, median, range) & $151.50(7.00,339.00)$ & $68.00(11.00,950.00)$ & 0.773 \\
\hline AST (U/L, median, range) & $62.00(7.00,259.00)$ & $53.50(13.00,883.00)$ & 0.695 \\
\hline Potassium (mM) & $4.26(2.70,5.66)$ & $4.25(3.36,5.55)$ & 0.643 \\
\hline Sodium (mM) & $138.10(132.00,143.90)$ & $141.15(135.90,147.50)$ & 0.003 \\
\hline Chloride (mM) & $98.35(92.00,106.10)$ & $99.60(93.70,107.70)$ & 0.143 \\
\hline Albumin $(g / L)$ & $35.35(28.30,47.50)$ & $32.40(22.70,42.60)$ & 0.101 \\
\hline BUN (mM) & $4.24(2.37,7.29)$ & $4.32(1.47,6.57)$ & 0.386 \\
\hline Creatinine $(\mu \mathrm{M})$ & $71.65(39.90,93.00)$ & $63.50(37.00,118.20)$ & 0.115 \\
\hline Total cholesterol (mM) & $5.32(2.80,8.88)$ & $4.08(2.34,6.00)$ & 0.002 \\
\hline Triglycerides (mM) & $2.28(0.51,9.38)$ & $1.69(0.62,4.32)$ & 0.334 \\
\hline HDL (mM) & $0.92(0.42,3.01)$ & $0.96(0.17,2.04)$ & 0.599 \\
\hline LDL (mM) & $2.41(0.91,4.30)$ & $1.97(0.36,2.68)$ & 0.045 \\
\hline Tumor stage & & & 0.655 \\
\hline I & $4(16.6 \%)$ & $2(8.3 \%)$ & \\
\hline II & $16(66.7 \%)$ & $19(79.2 \%)$ & \\
\hline III & $4(16.7 \%)$ & $3(12.5 \%)$ & \\
\hline ECOG & & & 0.855 \\
\hline 0 & $5(20.8 \%)$ & $7(29.2 \%)$ & \\
\hline 1 & $14(58.4 \%)$ & $13(54.2 \%)$ & \\
\hline 2 & $5(20.8 \%)$ & $4(16.6 \%)$ & \\
\hline Radiotherapy & 2 & 1 & $>0.99$ \\
\hline \multicolumn{4}{|l|}{ Chemotherapy } \\
\hline Gemcitabine + capecitabine & 1 & 3 & 0.609 \\
\hline Gemcitabine + oxaliplatin & 2 & 1 & $>0.99$ \\
\hline Gemcitabine + TGOPC & 2 & 1 & $>0.99$ \\
\hline Capecitabine + irinotecan & 1 & 0 & $>0.99$ \\
\hline Gemcitabine & 3 & 2 & $>0.99$ \\
\hline TGOPC & 0 & 1 & $>0.99$ \\
\hline P-gp & & & $<0.001$ \\
\hline$<85$ & $6(25.0 \%)$ & $18(75.0 \%)$ & \\
\hline$\geqslant 85$ & $18(75.0 \%)$ & $6(25.0 \%)$ & \\
\hline
\end{tabular}

Categorical data are reported as number (\%) and continuous data as median (range) [chi-squared test (categorical) and Student's $t$-test (continuous)]. *Defined as drug treatment for hypertension. N/A, not available; WBC, white blood cell; RBC: red blood cells; ALT, alanine aminotransferase; AST, aspartate aminotransferase; BUN, blood urea nitrogen; HDL, high-density lipoprotein cholesterol; LDL, lowdensity lipoprotein cholesterol; ECOG: Eastern Cooperative Oncology Group; P-gp: P-glycoprotein; TGOPC: tegafur gimeracil oteracil potassium capsule. 
median duration of DM was 3.0 (range, 0.1-30.0) years. As shown in Table 1, the blood levels of fasting blood glucose $(P=0.001)$, sodium $(P=0.003)$, total cholesterol $(P=0.002)$, and low-density lipoprotein cholesterol $(P=0.045)$ were significantly higher in the DM group than in the non-DM group, suggesting dysregulated glucose and lipid metabolism in patients with $\mathrm{PaC}$ and DM. In addition, the DM group included more women (58.3 vs $25.0 \%, \mathrm{P}=0.02$ ), and the patients in the non-DM group were taller $(P=0.03)$, without a difference in weight $(P=0.26)$. There were no differences between the two groups regarding smoking, alcohol, other biochemical indicators, tumor stage, and Eastern Cooperative Oncology Group (ECOG) score (all $P>0.05$ ). The proportion of patients with a $\mathrm{P}$-gp score $\geqslant 85$ was higher in the DM group than in the non-DM group (75.0 vs $25.0 \%$, $\mathrm{P}<0.001)$.

P-gp, but not CYP3A4, was significantly upregulated in duodenum tissues of patients with $\mathrm{PaC}$ and DM

Since P-gp and CYP3A4 are able to form an intestinal absorption barrier that is closely associated with multidrug resistance (17), we sought to investigate the possible association of P-gp and CYP3A4 expression with the presence of $\mathrm{DM}$ in patients with $\mathrm{PaC}$. Based on the $\mathrm{IHC}$ results for these two proteins in duodenum tissue samples of patients with $\mathrm{PaC}$ with/without DM (Figure 1), the mean IHC score of duodenal P-gp in the DM group was significantly higher than that in the non-DM group (Table 2; Figure $2 \mathrm{~A}-\mathrm{F}$ ), whereas the mean IHC score of duodenal CYP3A4 was not significantly different between the two groups (Table 2; Figure 3A-F).

\section{DM and duodenal P-gp levels were not associated with the OS of patients with $\mathrm{PaC}$}

The OS of patients with $\mathrm{PaC}$ and DM or high expression of P-gp (IHC score > 85) was not significantly lower compared with that of patients without DM or with low expression of $\mathrm{P}$-gp $(\mathrm{P}=0.291$ and $\mathrm{P}=0.958)$ (Figure 4). In addition, the multivariable Cox regression analysis showed that there was no association between other variables and survival in patients with $\mathrm{PaC}$ (data not shown). These data suggested that, at least in this cohort of patients, the presence of DM and high duodenal P-gp levels were not associated with the $\mathrm{OS}$ of patients with $\mathrm{PaC}$.
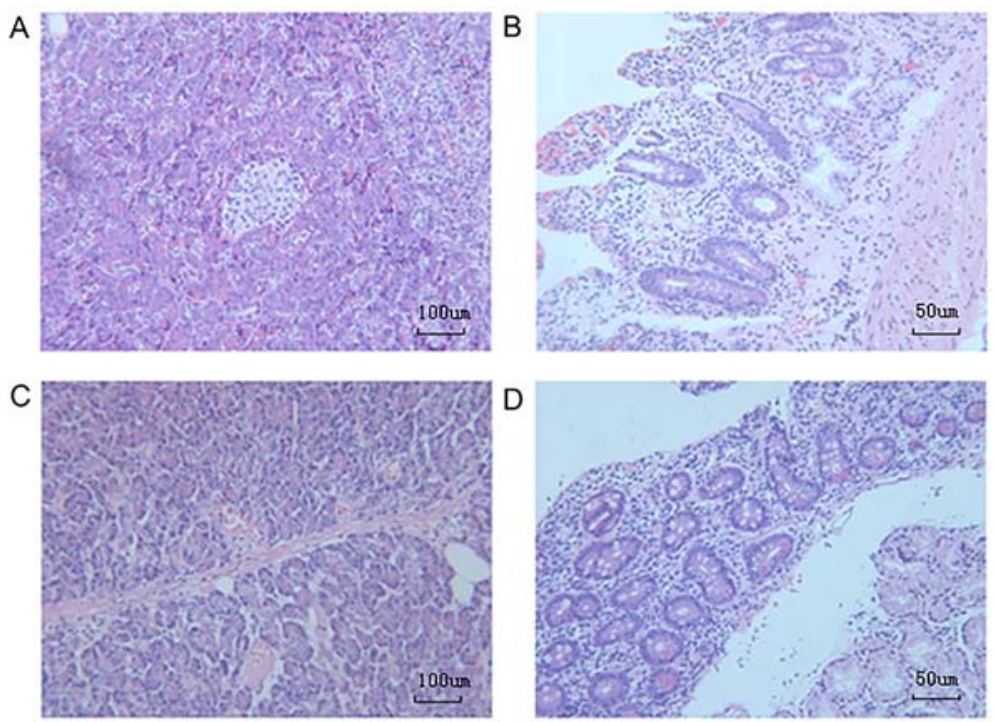

Figure 1. Hematoxylin and eosin staining of duodenum tissues from patients with pancreatic cancer with (A and B) or without (C and D) diabetes mellitus. Representative images are shown. Magnification $100 \times(\mathbf{A}$ and $\mathbf{C}$, scale bar $100 \mu \mathrm{m})$ and $200 \times(\mathbf{B}$ and $\mathbf{D}$, scale bar $50 \mu \mathrm{m})$.

Table 2. Duodenal expression score (1-100) of CYP3A4 and P-gp proteins in patients with pancreatic cancer with or without diabetes mellitus (DM).

\begin{tabular}{lcrr}
\hline & DM $(n=24)$ & Non-DM $(n=24)$ & $P$ \\
\hline P-gp & $92.50(20.00,100.00)$ & $65.00(10.00,95.00)$ & $<0.001$ \\
CYP3A4 & $80.00(20.00,100.00)$ & $72.50(0.00,100.00)$ & 0.312 \\
\hline
\end{tabular}

Data are reported as median (range) (Student's t-test). CYP3A4: cytochrome P450 3A4; P-gp: P-glycoprotein. 


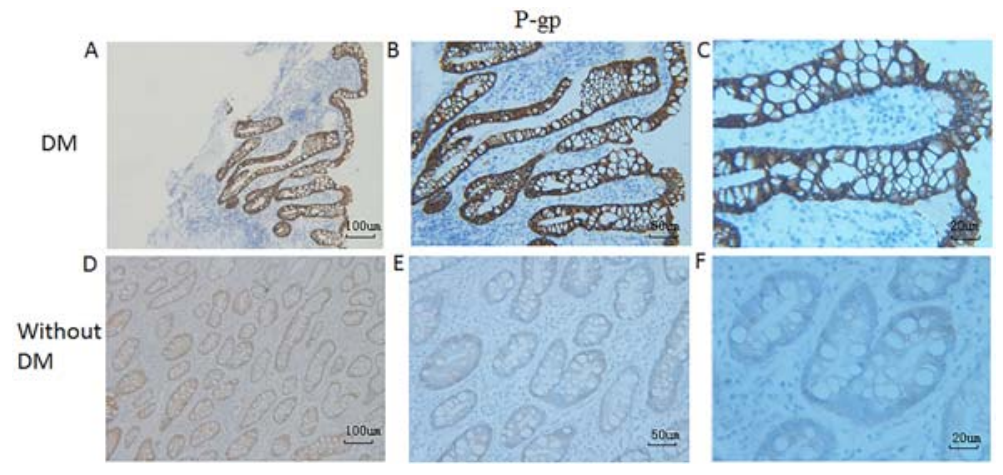

Figure 2. Immunohistochemical staining of $P$-glycoprotein (P-gp) in duodenum tissues of patients with pancreatic cancer with (A, B, and C) or without (D, E, and F) diabetes mellitus (DM). Representative images are shown. Magnification $100 \times(\mathbf{A}$ and $\mathbf{D}$, scale bar $100 \mu \mathrm{m})$, $200 \times(\mathbf{B}$ and $\mathbf{E}$, scale bar $50 \mu \mathrm{m})$, or $400 \times(\mathbf{C}$ and $\mathbf{F}$, scale bar $20 \mu \mathrm{m})$.

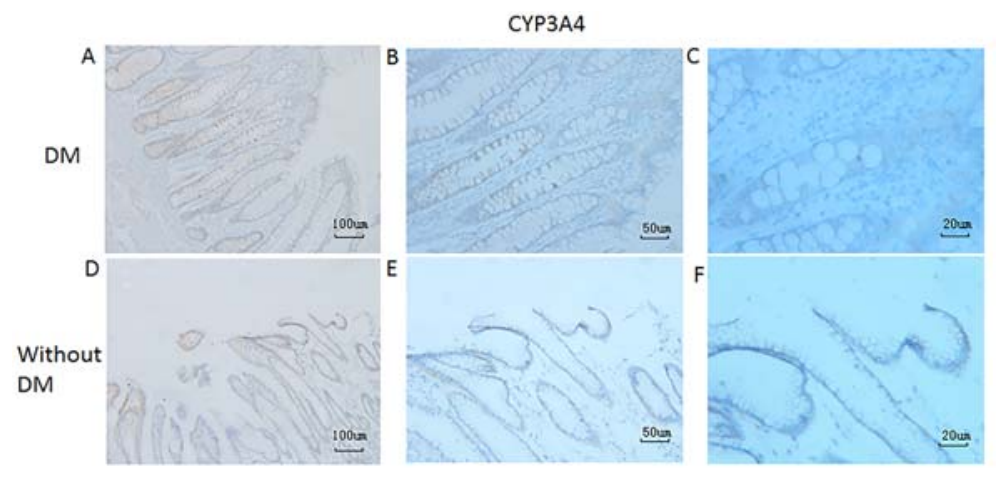

Figure 3. Immunohistochemical staining of cytochrome P450 3A4 (CYP3A4) in duodenum tissues of patients with pancreatic cancer with $(\mathbf{A}, \mathbf{B}$, and $\mathbf{C})$ or without $(\mathbf{D}, \mathbf{E}$, and $\mathbf{F})$ diabetes mellitus $(\mathrm{DM})$. Representative images are shown. Magnification $100 \times(\mathbf{A}$ and $\mathbf{D}$, scale bar $100 \mu \mathrm{m}), 200 \times(\mathbf{B}$ and $\mathbf{E}$, scale bar $50 \mu \mathrm{m})$, or $400 \times(\mathbf{C}$ and $\mathbf{F}$, scale bar $20 \mu \mathrm{m})$.

\section{Cox analysis for death}

The univariate and multivariate Cox analyses were conducted. Triglycerides $(\mathrm{HR}=0777,95 \% \mathrm{Cl}: 0.616-0.980$, $\mathrm{P}=0.033)$ and $\mathrm{ECOG}=2(\mathrm{HR}=3.047,95 \% \mathrm{Cl}: 1.098-8.459$, $\mathrm{P}=0.032)$ were associated with mortality in the univariate analyses. In the multivariate analysis, no variable was associated with mortality.

\section{Discussion}

In the present study, we compared the protein levels of P-gp and CYP3A4 in duodenum tissues between patients with $\mathrm{PaC}$ with/without $\mathrm{DM}$ and found that $\mathrm{P}-\mathrm{gp}$, but not CYP3A4, was significantly upregulated in the DM group vs the non-DM group, suggesting a possible positive correlation between duodenal expression of P-gp and the presence of $\mathrm{DM}$ in patients with $\mathrm{PaC}$. Despite the negative results of the association analyses of patient survival with DM and duodenal P-gp levels, our findings may provide new clues for a better understanding of the chemoresistance-related genetic alterations in $\mathrm{PaC}$ in the context of DM.
P-gp protein expression was different in the DM and non-DM groups, suggesting that the expression of the $\mathrm{P}$-gp protein was affected by DM and that drug resistance might theoretically be different. Although P-gp was first identified in drug-resistant cancer cells, it is also expressed in a wide variety of normal tissues (small intestine, liver, and kidney) as well as in blood-tissue barriers (23), serving as an efflux transporter against the entry of toxic xenobiotics, such as therapeutic drugs, dietary compounds, and environmental toxins, into the tissues (24). Previous studies suggest that the expression of P-gp changes in various pathological conditions at different tissue levels $(25,26)$. With reference to DM, Yeh et al. $(25)$ reported that hyperglycemia suppresses renal P-gp expression in rats, whereas another group has observed the overexpression of P-gp in the blood-brain barrier of streptozotocin-induced diabetic rats (27). As peroral bioavailability of drugs will be primarily affected by intestinal P-gp and patients with $\mathrm{PaC}$ and DM are usually prescribed multiple medications, it is of great importance to evaluate the intestinal P-gp expression in these patients. In this study, the upregulation of duodenal $\mathrm{P}$-gp expression in patients with $\mathrm{PaC}$ and DM was higher 

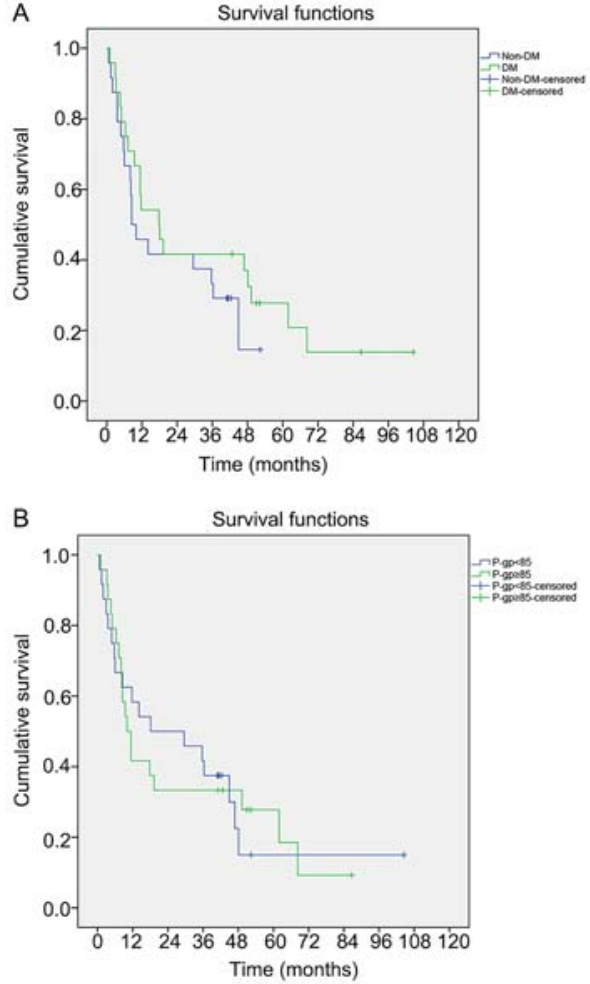

Figure 4. Kaplan-Meier analysis of survival time in patients with pancreatic cancer with or without diabetes mellitus (DM) $(P=0.284)(A)$ and duodenal $P$-glycoprotein $(P$-gp) score $<$ or $\geqslant 85(P=0.981)(B)$.

than that in patients with $\mathrm{PaC}$ but without $\mathrm{DM}$, which was consistent with a recent study showing that intestinal P-gp can be significantly increased along with the progression of DM in rats (21). As an ATP-mediated transporter, the ATPase enzyme is required for the function of P-gp (28), but we did not measure the ATPase activity in this retrospective cohort study, which should be addressed in the future.

Elevated expression of P-gp in response to chemotherapy has been reported in different malignancies, and $\mathrm{P}$-gp was first identified in chemotherapy-resistant cancer cells. For example, a dramatic increase in P-gp expression from 15 to $43 \%$ was observed in breast tumor biopsies following treatment with conventional chemotherapy (29). Likewise, in a multiple myeloma study, P-gp expression was $6 \%$ at diagnosis, which increased to $43 \%$ after treatment (30). In addition, the expression of P-gp is associated with the survival of patients with cancer. Indeed, a shorter OS in patients with acute myelogenous leukemia was associated with upregulated P-gp expression (31). Similarly, patients with $\mathrm{PaC}$ and high P-gp expression in $\mathrm{PaC}$ tissues have shorter survival compared with those with weak or moderate expression of P-gp (32).
Paradoxically, in the same report, it was also observed that the survival of patients with high expression of P-gp was not significantly different from that of those without detectable P-gp expression (32).

Therefore, based on the association between duodenal $\mathrm{P}$-gp levels and DM in patients with $\mathrm{PaC}$, we explored whether DM or duodenal P-gp expression could affect the survival of patients with $\mathrm{PaC}$. We observed shorter survival in the DM and high P-gp expression groups compared with the non-DM and low P-gp expression groups, but the differences between the groups were not significant, possibly due to the small sample size of this study and the impossibility of adjusting for confounding factors. Furthermore, most of the patients in this study were treated with metformin, the first-line drug for type-2 diabetes (33). In addition to its hypoglycemic effects, metformin has been shown to inhibit chemotherapy resistance in a variety of solid tumors, including $\mathrm{PaC}$ (34-36). The mechanisms include decreased microvessel density, leakage, and hypoxia (34), enhancement of the effects of anti-proliferation drugs (35), and inhibition of chemoresistance (36). Those effects could improve the survival of the patients and might have contributed to the lack of a significant difference between the DM and non-DM groups in the present study. Indeed, metformin is associated with improved survival in patients with solid tumors (37-40). Future studies should seek to include patients with $\mathrm{PaC}$ and $\mathrm{DM}$ but without treatment for DM with metformin.

The primary limitation of the present study is that it had a small sample size from a single center. In addition, it was a retrospective cohort study, resulting in selection biases. The data that could be analyzed were limited to those available from the medical charts. Furthermore, based on the available data, it was impossible to determine the causal relationship between $\mathrm{DM}$ and $\mathrm{PaC}$ with any certitude. Thus, the prognostic value of DM and P-gp in $\mathrm{PaC}$ remains disputable (11-13), and a larger sample size may be required for further confirmation of the results of the present study.

In conclusion, our study showed that the protein levels of duodenal P-gp were significantly elevated in $\mathrm{PaC}$ patients with DM compared with those without DM. Although there was no significant difference between patient survival and DM or P-gp levels, our results may still be helpful for a better understanding of drug resistancerelated gene dysregulation in $\mathrm{PaC}$ patients.

\section{Acknowledgments}

This study was funded by the National Science Foundation of China (No. 81503136), the Changzhou HighLevel Medical Talents Training Project (No. 2016CZBJ010), the Changzhou Science and Technology Project (Applied Based Research No. 2016404), and the Jiangsu Pharmaceutical Association-Aosaikang Clinical Pharmacy Foundation (No. A201920). 


\section{References}

1. Bray F, Ferlay J, Soerjomataram I, Siegel RL, Torre LA, Jemal A. Global cancer statistics 2018: GLOBOCAN estimates of incidence and mortality worldwide for 36 cancers in 185 countries. CA Cancer J Clin 2018; 68: 394-424, doi: 10.3322/caac.21492.

2. Colbert LE, Hall WA, Nickleach D, Switchenko J, Kooby DA, Liu Y, et al. Chemoradiation therapy sequencing for resected pancreatic adenocarcinoma in the National Cancer Data Base. Cancer 2014; 120: 499-506, doi: 10.1002/cncr.28530.

3. Siegel R, Ma J, Zou Z, Jemal A. Cancer statistics, 2014. CA Cancer J Clin 2014; 64: 9-29, doi: 10.3322/caac.21208.

4. Hidalgo M, Cascinu S, Kleeff J, Labianca R, Lohr JM, Neoptolemos J, et al. Addressing the challenges of pancreatic cancer: future directions for improving outcomes. Pancreatology 2015; 15: 8-18, doi: 10.1016/j.pan.2014.10. 001.

5. Andren-Sandberg A. Prognostic factors in pancreatic cancer. N Am J Med Sci 2012; 4: 9-12, doi: 10.4103/ 1947-2714.92893.

6. Valsangkar NP, Bush DM, Michaelson JS, Ferrone CR, Wargo JA, Lillemoe KD, et al. N0/N1, PNL, or LNR? The effect of lymph node number on accurate survival prediction in pancreatic ductal adenocarcinoma. $J$ Gastrointest Surg 2013; 17: 257-266, doi: 10.1007/s11605-012-1974-7.

7. American Diabetes A. Diagnosis and classification of diabetes mellitus. Diabetes Care 2009; 32: S62-S67, doi: 10.2337/dc09-S062.

8. Guariguata L, Whiting DR, Hambleton I, Beagley J, Linnenkamp U, Shaw JE. Global estimates of diabetes prevalence for 2013 and projections for 2035. Diabetes Res Clin Pract 2014; 103: 137-149, doi: 10.1016/j.diabres.2013. 11.002.

9. Ben $Q, X u$ M, Ning $X$, Liu J, Hong S, Huang W, et al. Diabetes mellitus and risk of pancreatic cancer: a metaanalysis of cohort studies. Eur J Cancer 2011; 47: 19281937, doi: 10.1016/j.ejca.2011.03.003.

10. Li D, Tang H, Hassan MM, Holly EA, Bracci PM, Silverman DT. Diabetes and risk of pancreatic cancer: a pooled analysis of three large case-control studies. Cancer Causes Control 2011; 22: 189-197, doi: 10.1007/s10552-010-9686-3.

11. Barone BB, Yeh HC, Snyder CF, Peairs KS, Stein KB, Derr $\mathrm{RL}$, et al. Long-term all-cause mortality in cancer patients with preexisting diabetes mellitus: a systematic review and meta-analysis. JAMA 2008; 300: 2754-2764, doi: 10.1001/ jama.2008.824.

12. Kim TD, Oh HJ, Kim KH, Kim SM, Kim JH, Jang BI, et al. Clinical characteristics of pancreatic cancer according to the presence of diabetes mellitus [in Korean]. Korean J Gastroenterol 2004; 43: 35-40, doi: 10.4166/kjg.2012.59.1.35.

13. Kang SP, Saif MW. Clinical outcome of pancreatic cancer patients with diabetes mellitus: is diabetes a poor prognostic factor? Highlights from the "2010 ASCO Annual Meeting". Chicago, IL, USA. June 4-8, 2010. JOP 2010; 11: 334-335, doi: $10.6092 / 1590-8577 / 3617$.

14. Lee HS, Park SW. Systemic chemotherapy in advanced pancreatic cancer. Gut Liver 2016; 10: 340-347, doi: $10.5009 / g n 15465$.
15. Whatcott CJ, Posner RG, Von Hoff DD, Han H. Desmoplasia and chemoresistance in pancreatic cancer. In: Grippo PJ, Munshi HG, editors. Pancreatic Cancer and Tumor Microenvironment. Trivandrum (India) 2012.

16. Eagling VA, Profit L, Back DJ. Inhibition of the CYP3A4mediated metabolism and P-glycoprotein-mediated transport of the HIV-1 protease inhibitor saquinavir by grapefruit juice components. Br J Clin Pharmacol 1999; 48: 543-552, doi: 10.1046/j.1365-2125.1999.00052.x.

17. Pal D, Kwatra D, Minocha M, Paturi DK, Budda B, Mitra AK. Efflux transporters- and cytochrome P-450-mediated interactions between drugs of abuse and antiretrovirals. Life Sci 2011; 88: 959-971, doi: 10.1016/j.lfs.2010.09.012.

18. Zajchowski DA, Karlan BY, Shawver LK. Treatment-related protein biomarker expression differs between primary and recurrent ovarian carcinomas. Mol Cancer Ther 2012; 11: 492-502, doi: 10.1158/1535-7163.MCT-11-0746.

19. Floriano-Sanchez E, Rodriguez NC, Bandala C, CoballaseUrrutia E, Lopez-Cruz J. CYP3A4 expression in breast cancer and its association with risk factors in Mexican women. Asian Pac J Cancer Prev 2014; 15: 3805-3809, doi: 10.7314/APJCP.2014.15.8.3805.

20. May M, Schindler C. Clinically and pharmacologically relevant interactions of antidiabetic drugs. Ther Adv Endocrinol Metab 2016; 7: 69-83, doi: 10.1177/204201881663 8050.

21. Dash RP, Ellendula B, Agarwal M, Nivsarkar M. Increased intestinal P-glycoprotein expression and activity with progression of diabetes and its modulation by epigallocatechin3-gallate: Evidence from pharmacokinetic studies. Eur $\mathrm{J}$ Pharmacol 2015; 767: 67-76, doi: 10.1016/j.ejphar.2015. 10.009 .

22. Borges-Ferro A, Santos AB, Filipe JL. Immunohistochemistry in formalin-gel fixed tissues. J Histotechnol 2014; 37: 48-53, doi: 10.1179/2046023614Y.0000000040.

23. Fromm MF. Importance of P-glycoprotein at blood-tissue barriers. Trends Pharmacol Sci 2004; 25: 423-429, doi: 10.1016/j.tips.2004.06.002.

24. Zhou SF. Structure, function and regulation of P-glycoprotein and its clinical relevance in drug disposition. Xenobiotica 2008; 38: 802-832, doi: 10.1080/00498250701867889.

25. Yeh SY, Pan HJ, Lin CC, Kao YH, Chen YH, Lin CJ. Hyperglycemia induced down-regulation of renal P-glycoprotein expression. Eur J Pharmacol 2012; 690: 42-50, doi: 10.1016/j.ejphar.2012.06.013.

26. Waghray $D$, Zhang $Q$. Inhibit or evade multidrug resistance p-glycoprotein in cancer treatment. J Med Chem 2018; 61: 5108-5121, doi: 10.1021/acs.jmedchem.7b01457.

27. Maeng HJ, Kim MH, Jin HE, Shin SM, Tsuruo T, Kim SG, et al. Functional induction of P-glycoprotein in the bloodbrain barrier of streptozotocin-induced diabetic rats: evidence for the involvement of nuclear factor-kappaB, a nitrosative stress-sensitive transcription factor, in the regulation. Drug Metab Dispos 2007; 35: 1996-2005, doi: 10.1124/ dmd.107.015800.

28. Varma MV, Ashokraj Y, Dey CS, Panchagnula R. P-glycoprotein inhibitors and their screening: a perspective 
from bioavailability enhancement. Pharmacol Res 2003; 48: 347-359, doi: 10.1016/S1043-6618(03)00158-0.

29. Chevillard S, Pouillart P, Beldjord C, Asselain B, Beuzeboc $P$, Magdelenat $H$, et al. Sequential assessment of multidrug resistance phenotype and measurement of S-phase fraction as predictive markers of breast cancer response to neoadjuvant chemotherapy. Cancer 1996; 77: 292-300, doi: 10.1002/(SICI)1097-0142(19960115)77:2<292::AID-CNCR $11>3.0 . \mathrm{CO} ; 2-\mathrm{X}$.

30. Grogan TM, Spier CM, Salmon SE, Matzner M, Rybski J, Weinstein RS, et al. P-glycoprotein expression in human plasma cell myeloma: correlation with prior chemotherapy. Blood 1993; 81: 490-495, doi: 10.1182/blood.V81.2.490. 490.

31. Zhou DC, Zittoun R, Marie JP. Expression of multidrug resistance-associated protein (MRP) and multidrug resistance (MDR1) genes in acute myeloid leukemia. Leukemia 1995; 9: 1661-1666.

32. Lu Z, Kleeff J, Shrikhande S, Zimmermann T, Korc M, Friess $\mathrm{H}$, et al. Expression of the multidrug-resistance 1 (MDR1) gene and prognosis in human pancreatic cancer. Pancreas 2000; 21: 240-247, doi: 10.1097/00006676-20001000000004.

33. Introduction: Standards of Medical Care in Diabetes-2020. Diabetes Care 2020; 43: S1-S2, doi: 10.2337/dc20-Sint.

34. Wang JC, Li GY, Wang B, Han SX, Sun X, Jiang YN, et al. Metformin inhibits metastatic breast cancer progression and improves chemosensitivity by inducing vessel normalization via PDGF-B downregulation. J Exp Clin Cancer Res 2019; 38: 235, doi: 10.1186/s13046-019-1211-2.

35. Candido S, Abrams SL, Steelman L, Lertpiriyapong K, Martelli AM, Cocco L, et al. Metformin influences drug sensitivity in pancreatic cancer cells. Adv Biol Regul 2018; 68: 13-30, doi: 10.1016/j.jbior.2018.02.002.

36. Bai M, Yang L, Liao $\mathrm{H}$, Liang X, Xie B, Xiong J, et al. Metformin sensitizes endometrial cancer cells to chemotherapy through IDH1-induced Nrf2 expression via an epigenetic mechanism. Oncogene 2018; 37: 5666-5681, doi: 10.1038/ s41388-018-0360-7.

37. Zi F, Zi H, Li Y, He J, Shi Q, Cai Z. Metformin and cancer: An existing drug for cancer prevention and therapy. Oncol Lett 2018; 15: 683-690, doi: 10.3892/ol.2017.7412.

38. Saraei P, Asadi I, Kakar MA, Moradi-Kor N. The beneficial effects of metformin on cancer prevention and therapy: a comprehensive review of recent advances. Cancer Manag Res 2019; 11: 3295-3313, doi: 10.2147/CMAR.S200059

39. Yu H, Zhong X, Gao P, Shi J, Wu Z, Guo Z, et al. The potential effect of metformin on cancer: an umbrella review. Front Endocrinol (Lausanne) 2019; 10: 617.

40. Lee DJ, McMullen CP, Foreman A, Huang SH, Lu L, Xu W, et al. Impact of metformin on disease control and survival in patients with head and neck cancer: a retrospective cohort study. J Otolaryngol Head Neck Surg 2019; 48: 34, doi: 10.1186/s40463-019-0348-5. 